\title{
Molecular cloning and tissue distribution of a Schistosoma japonicum gene encoding AMY-1
}

\author{
DONG HU ${ }^{1}$, JING WU $^{1}$, XIAOPING TANG ${ }^{2}$, FENGYU HU $^{2}$, YABO YANG $^{3}$, \\ JIUWEI DU ${ }^{1}$, SONG $\mathrm{YE}^{1}$ and RONGBO ZHANG ${ }^{1}$ \\ ${ }^{1}$ Department of Medical Immunology, Medical School, Anhui University of Science and Technology, Huainan 232001; \\ ${ }^{2}$ Institute of Infectious Disease, Guangzhou No.8 People's Hospital, Guangzhou 510050; \\ ${ }^{3}$ The Second Affiliated Hospital Of Sun Yat-sen University, Guangzhou 510120, P.R. China
}

Received May 10, 2011; Accepted August 12, 2011

DOI: $10.3892 / \mathrm{mmr} .2011 .565$

\begin{abstract}
It is well known that the mammalian associate of Myc-1 (AMY-1) plays a significant role in spermatogenesis or cellular differentiation. A full-length complementary DNA (cDNA) encoding AMY-1 of Schistosoma japonicum (SjAMY-1) was identified and isolated from a cDNA library. The gene contained an open reading frame of 315 nucleotides, encoding 105 amino acids. Sequence analysis showed that SjAMY-1 shares $65.7 \%$ homology with Homo sapiens AMY-1 amino acids and contains a conserved domain from the AMY-1 family. In this study, we cloned and expressed a recombinant SjAMY-1 (rSjAMY-1) with a molecular size of $14 \mathrm{kDa}$. The native SjAMY-1 in soluble worm antigen was identified by anti-rSjAMY-1 sera in the Western blot analysis, which demonstrated the presence of this protein in the parasite. Immunofluorescence studies indicated a localization of SjAMY-1 in various tissues and organs including the tegument and subtegumental muscles in adult worms, the ventral sucker in cercariae and the internal structures of eggs. Given the key roles of mammalian AMY-1 in cell proliferation and differentiation, the characterization of SjAMY-1 may allow for a better understanding of the development of S.japonicum.
\end{abstract}

\section{Introduction}

Schistosoma japonicum (S. japonicum) is a blood-dwelling trematode of the genus Schistosoma, and is responsible for the serious but neglected human disease of schistosomiasis (1). Schistosoma have a complex life cycle involving distinct life cycle stages that are adapted for transmission between the aquatic snail and the mammalian hosts. Current attempts to

Correspondence to: Professor Rongbo Zhang and Dr Jing Wu, Department of Medical Immunology, Medical School, Anhui University of Science and Technology, No. 25 Middle Dongshan Road, Huainan, Anhui 232001, P.R. China

E-mail: lory456@126.com; wujing8008@126.com

Key words: Schistosoma japonicum, AMY-1, clone, expression, tegument, sucker, eggs control schistosomiasis depend solely on the repeated administration of the drug, praziquantel, which is effective against adult worms dwelling in the definitive hosts (2). However, with no replacements pending, a severe shortcoming of this drug is the developing resistance of the parasite (3). Therefore, efforts are now focused on identifying new targets against schistosomiasis.

Associate of Myc-1 (AMY-1) was originally identified as a c-Myc-binding protein that enhances c-Myc transcriptional activity (4). c-Myc was suggested to play key roles in cell proliferation, differentiation, transformation and apoptosis (5). AMY-1 mostly localizes in the cytoplasm of cells expressing c-myc at low levels, but translocates into the nucleus in the $\mathrm{S}$ phase of the cell cycle upon an increase of c-myc expression (4). In addition to a c-Myc-independent manner, AMY-1 plays other roles. Overexpression of AMY-1 alone triggers the differentiation of K562 cells to erythrocytes (5). AMY-1 is found in the mitochondria of somatic cells, and in sperm AMY-1 is found to play a role in spermatogenesis $(7,8)$. AMY-1 is an essential modulator of protein kinase-A (PKA) (9), and PKA signaling in Schistosoma mansoni is required for cercariae viability and may play a role in the reproductive activity of adult worms (2). However, the characterization of the AMY-1 homolog from S. japonicum remains to be elucidated.

In the present study, we identified an AMY-1 homolog (SjAMY-1) from S. japonicum, cloned and expressed the SjAMY-1 gene and indicated the protein localization in various tissues of the schistosoma.

\section{Materials and methods}

Parasites and animals. The Chinese strain of S. japonicum maintained in Oncomelania hupensis snails was purchased from the Jiangsu Provincial Institute of Parasitic Diseases, Wuxi, China. Parasites in various developmental stages, including eggs, cercariae and adults were prepared as described (1). Briefly, cercariae were released from 10 infected snails in a beaker containing $20 \mathrm{ml}$ of water under light for $2 \mathrm{~h}$ at room temperature. New Zealand white rabbits were percutaneously infected with $1000 \mathrm{~S}$. japonicum cercariae. Adult worms were obtained by perfusion of the infected rabbits. 
S. japonicum eggs were isolated from the livers of the infected rabbits. Animal procedures were approved by the Animal Experimentation Ethics Committee of Sun Yat-sen University.

Soluble worm antigen preparation (SWAP). The S. japonicum adults obtained by perfusion of infected rabbits were washed and suspended in phosphate-buffered saline (PBS) ( $\mathrm{pH}$ 7.4). Worms were homogenized in PBS containing $1 \mathrm{mM}$ phenylmethylsulfonyl fluoride and $2 \mu \mathrm{g} / \mathrm{ml}$ leupeptin, followed by sonication for $2 \mathrm{~min}$ on ice. The homogenate was centrifuged at $16,000 \mathrm{x} \mathrm{g}$ for $30 \mathrm{~min}$ at $4^{\circ} \mathrm{C}$. The supernatant was filtrated through a $0.22 \mu \mathrm{m}$ filter. Following quantification of the protein concentration using a Bradford kit, the SWAP was boiled for $5 \mathrm{~min}$ in 4X SDS loading buffer containing dithiothreitol, and stored at $-70^{\circ} \mathrm{C}$.

Identification and characterization of SjAMY-1. The amino acid sequence of Homo sapiens AMY-1 was used to search the $S$. japonicum GenBank by Tblastn algorithm. A UniGene (GenBank accession no: AY815350.1) with best similarity to Homo sapiens AMY-1 was identified. The Align X program of NTI Advance 10 software was used to perform sequence analysis and homology comparisons based on the deduced amino acid sequence.

Cloning, expression and purification of SjAMY-1. The full-length open reading frame (ORF) of the SjAMY-1 gene was amplified from complementary DNA (cDNA) of adult $S$. japonicum with specific primers (sense primer: AAACCA TGGAAATGTCATCCTACAAACCTG with one Nco I restriction site; antisense primer: CCGCTCGAGACTATT TCCATCATTTGTTC with one Xho I restriction site). The ORF of SjAMY-1 was then cloned into the E. coli expression vector pET28a(+). The recombinant plasmid was sequenced to ensure the authenticity of the cloned gene. For expression of the protein, the recombinant plasmid was transformed into E. coli BL21 (DE3) and induced by $1 \mathrm{mM}$ IPTG at $37^{\circ} \mathrm{C}$. The recombinant protein was expressed in bacteria as a soluble protein and was purified with His-Bind column chromatography (Novagen, Germany) under non-denaturing conditions, according to the manufacturer's instructions. The purified recombinant protein was confirmed by analysis of SDS polyacrylamide gel (SDS-PAGE).

Antiserum. For production of antiserum, the rats were immunized with $150 \mu \mathrm{g}$ purified $\mathrm{rSjAMY}-1$ protein mixed with an equal volume of complete Freund's adjuvant, followed by two boosts of $100 \mu \mathrm{g}$ rSjAMY-1 protein mixed with incomplete Freund's adjuvant at 2-week intervals. The antiserum was collected following the last immunization and stored at $-70^{\circ} \mathrm{C}$ until further use.

SDS-PAGE and Western blot analysis. Recombinant protein and SWAP were electrophoresed by SDS-PAGE. For the Western blot analysis, the proteins were electro-transferred from the gel onto a polyvinylidene difluoride membrane. Following blocking in 5\% skim milk in PBST (pH 7.4, 0.1\% Tween-20) at room temperature for $2 \mathrm{~h}$, the membrane was incubated with the diluted primary antibodies (sera from S. japonicum-infected rabbits, dilution 1:200; sera from rats immunized with rSjAMY-1, dilution 1:2000; sera from pre- immune rats, dilution 1:2000) for $2 \mathrm{~h}$, followed by incubation with the 4000 times diluted secondary antibodies including horseradish peroxidase-conjugated goat anti-rabbit $\operatorname{IgG}$ or rabbit anti-rat $\mathrm{IgG}$. The bands reacting to antibodies were visualized by diaminobenzidine reagents.

Immunolocalization by immunofluorescence. Adult worms and infected livers of rabbits were stained by immunofluorescence techniques as described by Yang et al (10). Adult worms and infected liver were fixed with $10 \%$ neutral formalin and embedded with paraffin and then sliced into sections. For staining cercariae, cercariae incubated in PBS containing $0.1 \%$ Triton X-100 for 10 min were placed on slides at room temperature until they dried, followed by fixation in acetone for $10 \mathrm{~min}$. The tissue sections were incubated in primary antibodies (sera from rats immunized with rSjAMY-1, 1:200; sera from pre-immune rats, 1: 200) for $2 \mathrm{~h}$ at $37^{\circ} \mathrm{C}$. This was followed by incubation in secondary Alexa Fluor 555-conjugated goat anti-rat $\mathrm{IgG}$ diluted 400 times for $1 \mathrm{~h}$ at $37^{\circ} \mathrm{C}$. The immunofluorescence was observed using a fluorescence microscope (Olympus IX61).

\section{Results}

Isolation and sequence analysis of SjAMY-1. The full length ORF of SjAMY-1 was amplified from the cDNA library of adults. Following sequencing, the ORF of 315 nucleotides encoding 105 amino acids was identified. Following the alignment of AMY-1 homologs, the SjAMY-1 protein showed $56.2 \%$ identity with a homolog of Ciona intestinalis and $65.7 \%$ homology with a homolog of Homo sapiens. The homologs from a number of species showed a conserved domain from the AMY-1 family in the alignment of the sequences, with the aa1-96 domain conserved in SjAMY-1 (Fig. 1).

Expression and purification of $r S j A M Y-1$. The ORF of sjamy-1 was cloned into the pET28a(+) vector and expressed with solubility in $E$. coli (DE3). The recombinant fusion protein was purified by nickel affinity chromatography under native conditions and analyzed with SDS-PAGE (Fig. 2). The rSjAMY-1 yield per 1 liter of culture amounts to $40 \mathrm{mg}$, as determined by the Bradford assay.

Analysis of anti-SjAMY-1 antibody in S. japonicum-infected rabbit serum. After 45 days, the sera were collected from rabbits infected with cercariae. The antibody titer of the sera was measured by ELISA (titer $>3200$ ) for immunoreactivity to SWAP (data not shown). With Western blot analysis, the rSjAMY-1 could not be probed by the infected sera of rabbits (Fig. 3). Accordingly, the sera of infected rabbits failed to recognize the native SjAMY-1 in SWAP. These results indicated that native SjAMY-1 may fail to induce circulating antibodies in infected rabbits.

The recognition of native and $r S j A M Y-1$ by anti-rSjAMY-1 sera. The anti-rSjAMY-1 sera were collected from rats immunized with the recombinant protein, and these rats developed specific antibodies to titers $>3.6 \times 10^{6}$, as determined by ELISA (data not shown). The native SjAMY-1 in SWAP and rSjAMY-1 were recognized by anti-rSjAMY-1 sera as shown 
1

60

Schistoma japonicum
Ciona intestinalis
Nematostella vectensis
Branchiostoma floridae
Xenopus tropicalis
Ornithorhynchus anatinus
Bos taurus
Homo sapiens
Monodephis domestica
Consensus
Schistoma japonicum
Ciona intestinalis
Nematostella vectensis
Branchiostoma floridae
Xenopus tropicalis
Ornithorhynchus anatinus
Bos taurus
Homo sapiens
Monodephis domestica
Consensus

(1) - ISSYKPG SKRE FRKYLEKSGVID LTKVLVGLYEEPEKP NALEFVIK HL OAEGPEI

(1) NIIIVYRAADSKRE FRKYLEKAGVID LTKVLVLYYEEPEKPNNALEFL HHL SGDGPET

(1) MTSOYRVADSKRE FRKYLEKAGVLDSLTKVLVLYEEPEKP NALDFVK HLHGCAPEI

(1) -VIIISYRAADSKRE FRKYLEKAGVD LTKVLVGLYEEPDKPNALDFLR HLGASSPEI

(1) - MANYKKADSKREQFRRYLEKAGVLDTLTKVLVALYEEPEKPNNALDFLK HIYGAAGPET

(1) - IAQYKKAADSKREQFRRYLEKSGVLDSLTKVLVALYEEPEKPN SALDFLKHHLGAAVPE

(1) -MAAYYKAADSKREQFRRYLEKSGVDTLTKVLVALYEEPEKPN SALDFL KHHLGAATPE

(1) -MAYYKKADSKREQFRRYLEKSGVLDTLTKVLVALYEEPEKPN SALDFLKHHLGMTPE

(1) - MAHYYKAADSKREQFRRYLEKSGVLDTLTKVLVALYEEPEKPN SALDFLKHILLASTPE

(1) MA YKAADSKREQFRRYLEKSGVLDTLTKVLVALYEEPEKPNNALDFLKHHLGAA PET

$61 \quad 107$

(60) SDVDTVKVIAELKOKLIEILESEN ELKRSINQOLIPGTGTNDGNS-

(60) PDVEALKVDVIELKAKVSQLDENIELKTRLEKYEPSVADDTAE--

(61) ADVDALKLEVSELROKVEDLIAEN DLKORLQTYEPQADEEVES---

(60) ADVEGLRLELSEARORVEQLSEENAELKAKLAQYEPPAEDHATES--

(60) ADVEALRLEVAELKOKYEAVLEENKELAKLAQHDPAOLIDEK SET

(60) PEIES IRLELAEMKEKYEAVLEENK LKTKLAQYEPPQEEKRAE---

(60) PEIELLRLELAEMKEKYEA IVEENK LKTKLAQYEPPQEEKRA E--

(60) PEIELLRLELAEMKEKYEAIVEENK LKAKLAQYEPPQEEKRAD---

(60) PEIELLRVELAETKKEKYEAVVEENK LKAKLAQYEPPQEEKRA E-

(61) PDVE LRLELAELK KYEALVEENKELK KLAQYEPPQEE E

Figure 1. Alignment of SjAMY-1 with the homologs of other species, including Ciona intestinalis (XP 002127905), Nematostella vectensis (XP 001634099), Branchiostoma floridae (XP 002604299), Xenopus tropicalis (NP 001017035), Ornithorhynchus anatinus (XP 001511674), Bos taurus (NP 001033654XP), Homo sapiens (CAG33217) and Monodelphis domestica (XP 001364995). The conserved domain of SjAMY-1 is underlined. The letters in white foreground and black background indicate the identical amino acid residues, white foreground and deep grey background are the conservative residues, and black foreground and white background are the non-similar residues.

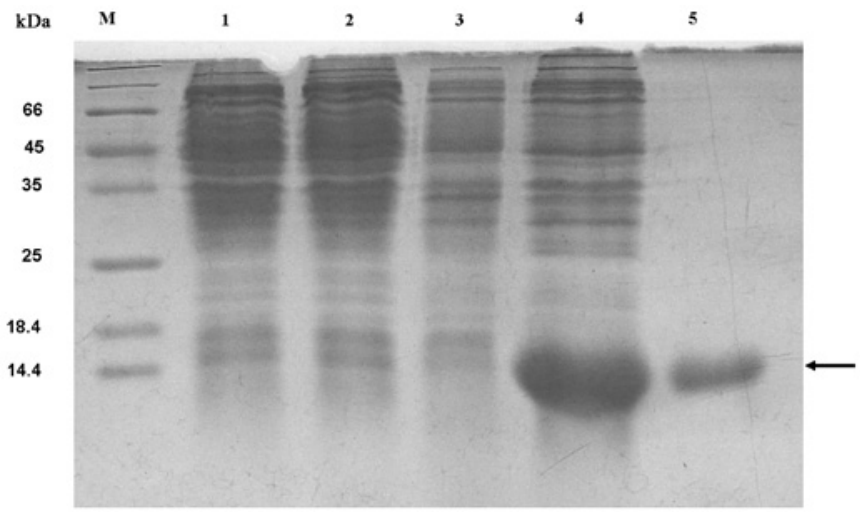

Figure 2. SDS-PAGE (15.0\%) analysis of rSjAMY-1. Lane M: molecular mass standards. Lane 1: total lysate of E. coli (DE3) containing pET28a prior to IPTG induction. Lane 2: total lysate of E. coli (DE3) containing pET28a following IPTG induction. Lane 3: total lysate of E. coli (DE3) containing pET28a-SjAMY-1 prior to IPTG induction. Lane 4: total lysate of E. coli (DE3) containing pET28a-SjAMY-1 following IPTG induction. Lane 5: fusion rSjAMY-1, purified by nickel-affinity chromatography, shown as the $14 \mathrm{kDa}$ band, marked by a black arrow.

in Fig. 3. Sera from pre-immune rats as a negative control did not identify the native SjAMY-1 or rSjAMY-1.

Immunolocalization of SjAMY-1 in S. japonicum. SjAMY-1 was probed by immunofluorescence in $S$. japonicum adults, cercariae and eggs. Fluorescence was observed in the tegument and subtegumental muscles of the adult worm (Fig. 4, panel A1). Immunofluorescence showed SjAMY-1 localized in the ventral sucker of the cercariae (Fig. 4, panel B1). The internal structures of the eggs were also stained with fluores-

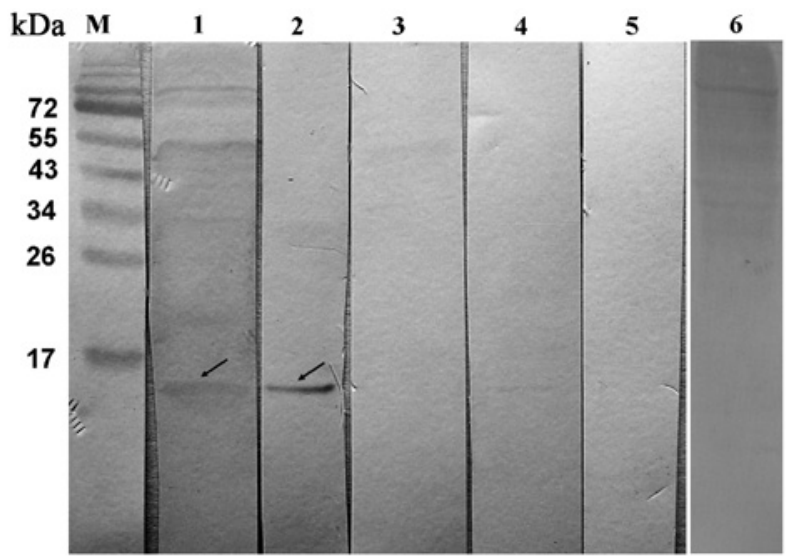

Figure 3. Western blot analysis of rSjAMY-1 and native SjAMY-1. Lane M: molecular mass standards. Lane 1: SWAP was probed with anti-rSjAMY-1 serum. Lane 2: rSjAMY-1 was probed with anti-rSjAMY-1 serum. Lane 3: SWAP was probed with the serum from pre-immune rats. Lane 4: rSjAMY-1 was probed with the serum from pre-immune rats. Lane 5: rSjAMY-1 was probed with the sera from $S$. japonicum-infected rabbits. Lane 6: SWAP was probed with the sera from $S$. japonicum-infected rabbits. The native SjAMY-1 (lane 1) and rSjAMY-1 probed by anti-rSjAMY-1 serum are marked by black arrows.

cence (Fig. 4, panel C1). Corresponding fluorescence was not observed as the primary antibody in negative controls with serum from pre-immune rats (Fig. 4, panel A2, B2 and C2).

\section{Discussion}

AMY-1 may be a significant candidate as a key regulating molecule in S. japonicum, since it is known to stimulate the 

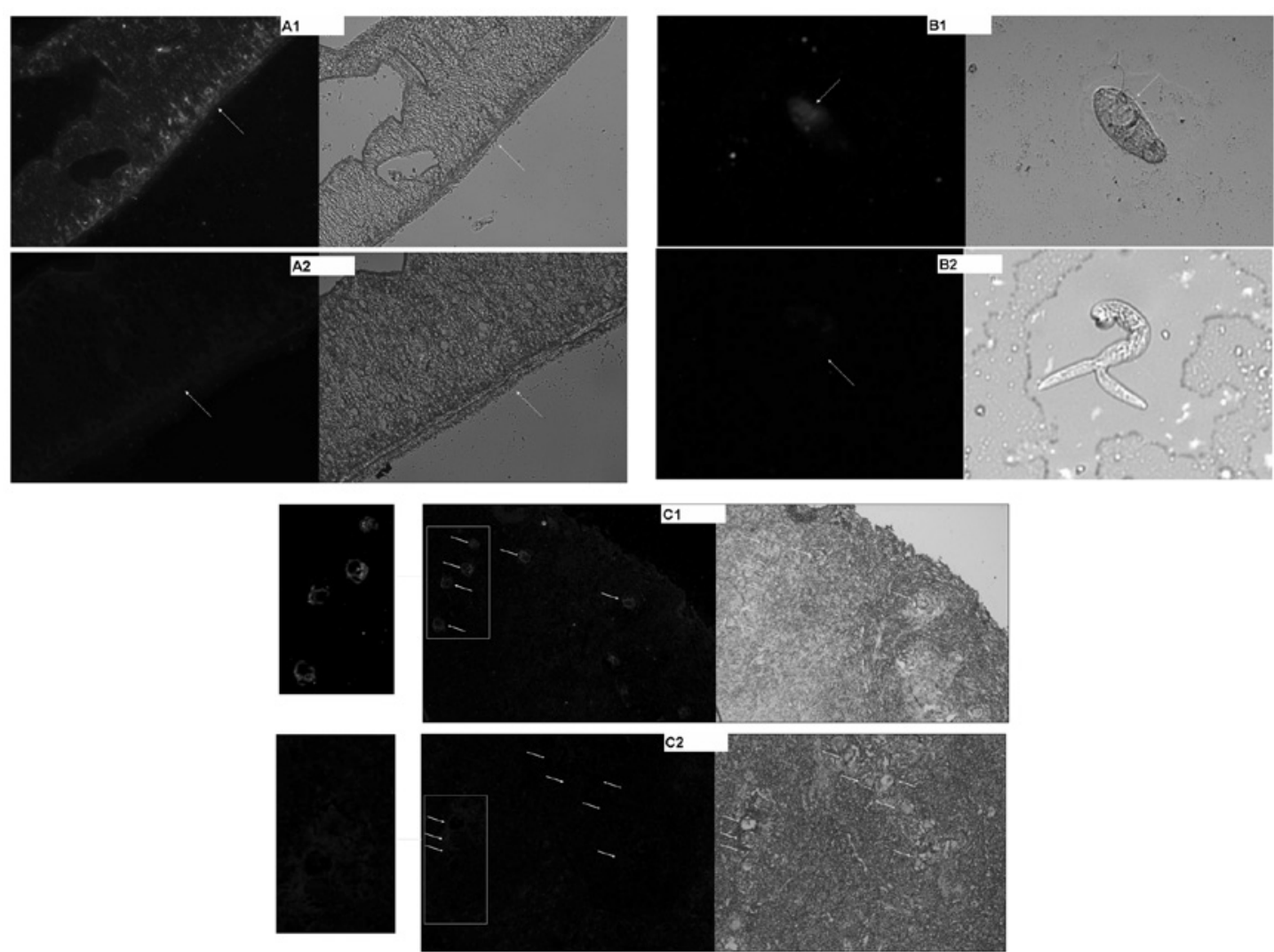

Figure 4. Immunolocalization of SjAMY-1 in S.japonicum. The adult and egg sections and cercariae were stained with anti-rSjAMY-1. A1: the tegument and subtegumental muscles of the adult worm were stained with anti-rSjAMY-1 serum. B1: the ventral sucker of cercariae was stained with anti-rSjAMY-1 serum. $\mathrm{C} 1$ : the eggs in the infected liver sections were stained with anti-rSjAMY-1 sera, right panel represents shows the magnified images of the eggs. A2, B2 and $\mathrm{C} 2$ are the controls with the serum from pre-immune rat.s The stained regions are marked by white arrows.

transcriptional activity of c-myc, trigger cell differentiation, be involved in spermatogenesis and modulate the PKA signal pathway in vertebrates (4-9). In this study, the aim was to identify the AMY-1 homolog of S. japonicum and explore its specific characteristics in various developmental stages.

In this study, we have cloned a homologous gene of AMY-1 from S. japonicum, and named it SjAMY-1. Sequence analysis with alignment of amino acid sequences revealed a high degree of homology between SjAMY-1 and other homologs of a number of species. Since SjAMY-1 has been shown to share similarities with AMY-1 of Homo sapiens, including protein sequences with $65.7 \%$ homology and a conserved domain of the AMY-1 family, it is likely to be the homolog of Homo sapiens AMY-1 (Fig. 1). SjAMY-1 may be a specific protein for $S$. japonicum since no significant homolog was found in Platyhelminthes. The characteristics of AMY-1 in phylogenesis may provide a possible explanation for the parasite's escape from the immune system of the host.

Western blot analysis verified the presence of SjAMY-1 in the adult worms. The native SjAMY-1 detected by anti-rSjAMY-1 serum required a component of soluble worm extract. The fact that the recombinant SjAMY-1 and the native molecule failed to be recognized by the sera of infected rabbits indicates that the soluble protein in the schistosoma may be unable to stimulate the production of the circulating antibodies in rabbits during infection. The manner in which SjAMY-1 escapes from the immune surveillance should be determined. SjAMY-1 was mainly localized in the subtegu- mental muscles and tegument of adults, which is crucial in protecting the parasite against the host immune system (Fig. 4). Immunofluorescence was also found in the ventral suckers of cercariae, by which the cercariae are able to attach to and penetrate into hosts and in the internal structures of the eggs, which are a significant cause of hepatic fibrosis (Fig. 4).

Considering that $S$.japonicum is a parasite with activities of invasion and powerful egg production, SjAMY-1 may be a new potential target for vaccines or chemotherapeutic agents against schistosomiasis. With the available recombinant protein and antibodies, more studies should be conducted to elucidate the exact role of SjAMY-1 in S.japonicum.

\section{Acknowledgements}

This study was supported by The National Nature Science Foundation of China (no. 81041083), Anhui Province Ministry of Education Key Projects (no. KJ2009A164), Colleges and Universities Education Project of Anhui Province (no. 2008jp1042) and the Key Scientific and Technology Projects of Guangzhou Health Bureau (no. 2009-ZDi-06).

\section{References}

1. Hu D, Wu J, Hu F, Yang Y, Liang C, Chen J, Wang L, Wang P, Wang $\mathrm{X}, \mathrm{Xu} \mathrm{J}, \mathrm{Hu} \mathrm{X}$ and $\mathrm{Yu} \mathrm{X}$ : Stage and tissue specific differences in SjBMI1, a Polycomb protein in Schistosoma japonicum. Parasitol Res 106: 677-682, 2010. 
2. Swierczewski BE and Davies SJ: Developmental regulation of protein kinase A expression and activity in Schistosoma mansoni: Int J Parasitol 40: 929-935, 2010.

3. Freitas TC, Jung E and Pearce EJ: TGF- $\beta$ signaling controls embryo development in the parasitic flatworm Schistosoma mansoni. PLoS Pathog 3: e52, 2007.

4. Taira T, Maeda J, Onishi T, Kitaura H, Yoshida S, Kato H, Ikeda M, Tamai K, Iquchi-Ariqa SM and Ariga H: AMY-1, a novel C-MYC binding protein that stimulates transcription activity of C-MYC. Genes Cells 3: 549-565, 1998.

5. Ryan KM and Birnie GD: Myc oncogenes: the enigmatic family. Biochem J 314: 713-721, 1996.

6. Furusawa M, Onishi T, Taira T, Iguchi-Ariga SM and Ariga $\mathrm{H}$ : AMY-1 is a trigger for the erythrocyte differentiation of K562 cells. Int J Oncol 16: 339-345, 2000.

7. Yukitake H, Furusawa M, Taira T, Iguchi-Ariga SM and Ariga H: AAT-1, a novel testis-specific AMY-1-binding protein forms a quaternary complex with AMY-1, A-kinase anchor protein 84 , and a regulatory subunit of cAMP-dependent protein kinase and is phosphorylated by its kinase. J Biol Chem 277: 45480-45492, 2002.
8. Yukitake H, Furusawa M, Taira T, Iguchi-Ariga SM and Ariga $\mathrm{H}$ : AMAP-1, a novel testis-specific AMY-1-binding protein, is differentially expressed during the course of spermatogenesis. Biochim Biophys Acta 1577: 126-132, 2002.

9. Furusawa M, Taira T, Iguchi-Ariga SM and Ariga H: AMY-1 interacts with S-AKAP84 and AKAP95 in the cytoplasm and the nucleus, respectively, and inhibits cAMP-dependent protein kinase activity by preventing binding of its catalytic subunit to A-kinase-anchoring protein (AKAP) complex. J Biol Chem 277:50885-50892, 2002 .

10. Yang Y, Hu D, Wang L, et al: Molecular cloning and characterization of a novel serpin gene of Clonorchis sinensis, highly expressed in the stage of metacercaria. Parasitol Rles 106 221-225. 2009 